\title{
Atypical Obstructive Pseudotumors in AIDS as the Initial Manifestation of Gastrointestinal Histoplasmosis
}

\author{
Alejandro Hallo ${ }^{1}$, Malena Camacho ${ }^{2}$, Alejandra Rojas ${ }^{3}$, Andres Mayancela ${ }^{4}$, Alberto Castillo II ${ }^{2}$ \\ 1. Internal Medicine, Hospital de Especialidad Eugenio Espejo, Quito, ECU 2. Internal Medicine, Hospital de \\ Especialidades Eugenio Espejo, Quito, ECU 3. Investigacion, Universidad Central del Ecuador, Quito, ECU 4. Internal \\ Medicine, Hospital Eugenio Espejo, Quito, ECU
}

Corresponding author: Alejandro Hallo, alejo_h1712@hotmail.com

\begin{abstract}
Histoplasma capsulatum is a dysmorphic fungus distributed worldwide commonly associated with pulmonary histoplasmosis. We report the case of an unusual presentation of gastrointestinal histoplasmosis leading to the obstruction of the intestinal lumen in a 30-year-old female, HIV positive, admitted to the hospital due to chronic abdominal pain and constipation. An initial abdominal CT revealed a mass in the sigmoid colon. A further colonoscopy showed an infiltrating, friable mass obstructing $80 \%$ of the lumen staining positive for H. capsulatum. The unspecific nature of the patient's symptoms along with the unusual presentation of the infection raises awareness about the importance of including new pathologies to differential diagnoses when treating AIDS patients.
\end{abstract}

Categories: Internal Medicine, HIV/AIDS, Infectious Disease

Keywords: aids, histoplasmosis, opportunistic mycoses

\section{Introduction}

Histoplasma capsulatum is one of the most common opportunistic pathogens affecting AIDS patients with a CD4 <100 [1]. Histoplasmosis results from impaired infection control from macrophages and CD4 [2]. Throughout the years, the use of antiretroviral therapy (ART) has modified the symptoms, location, and evolution of this disease as well as many other infections. Moreover, many AIDS-related infectious diagnoses occurred incidentally due to their often unspecific symptoms [3].

We report a case of a patient with a CD4 count of 64 cells/mL with chronic constipation from gastrointestinal histoplasmosis causing a pseudotumor obstructing $80 \%$ of the intestinal lumen.

Received 04/08/2020

Review began 04/13/2020 Review ended 04/15/2020 Published 04/22/2020

○ Copyright 2020 Hallo et al. This is an open access article distributed under the terms of the Creative Commons Attribution License CC-BY 4.0., which permits unrestricted use, distribution, and reproduction in any medium, provided the original author and source are credited.

\section{Case Presentation}

A 30-year-old cachectic woman with a past medical history of HIV and poor compliance with ART came to the ED due to diffuse abdominal pain and low-grade fever.

Physical examination revealed diffuse abdominal pain without rebound tenderness, jaundice, or palpable liver. The patient reported chronic constipation and weight loss $(12 \mathrm{~kg})$ over one year before the admission and denied respiratory symptoms.

Initial laboratory showed microcytic anemia (Hb 6.5, Hct 20\%, MCV 67 fL), CD4 count: 64 cells, viral load: 20 copies/mL, total proteins: 4.28 , albumin: 1.21 . Fecal occult blood was positive and no ova or parasites were seen in the stool sample.

An abdominal CT showed thickening of the intestinal wall and mesenteric lymphadenopathy.

An upper digestive endoscopy was performed due to the patient's CT results showing erythematous gastric mucosa in the antrum. Later, a colonoscopy revealed a friable pseudo tumoral mass in the sigmoid colon obstructing $80 \%$ of the lumen (Figure 1 ). 


\section{Cureus}
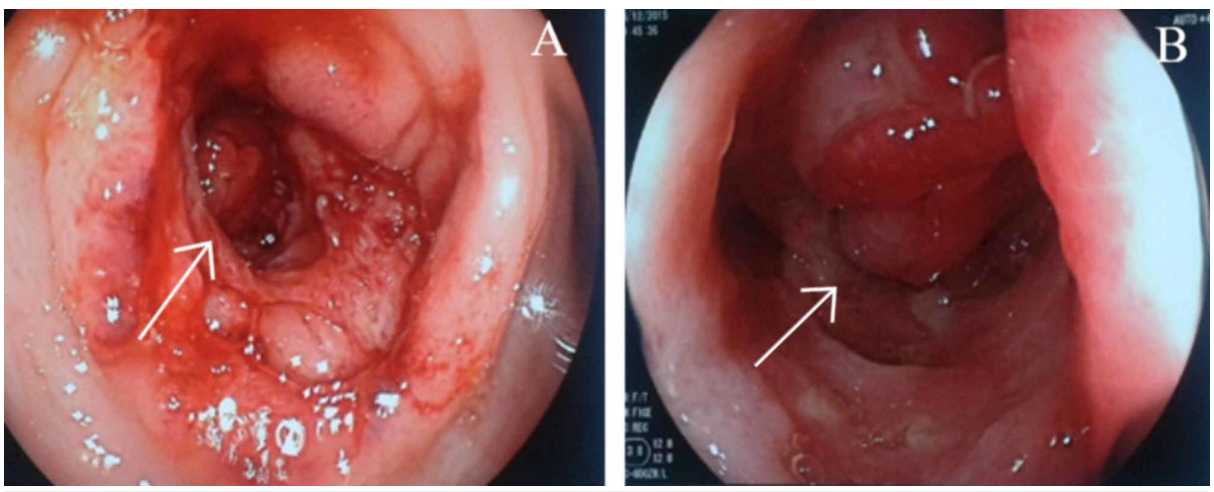

FIGURE 1: Sigmoid colon biopsy.

Ulcerated, irregular, friable, deep lesion with congestive edges, causing obstruction of $80 \%$ of the intestinal lumen.

Empirical treatment for tuberculosis (TB) was started as the patient came from an endemic area.

The histopathological examination of the pseudotumoral mass identified H. capsulatum (Figure 2). Therefore, the anti-TB treatment was replaced by amphotericin B $25 \mathrm{mg}$ daily for 25 days.

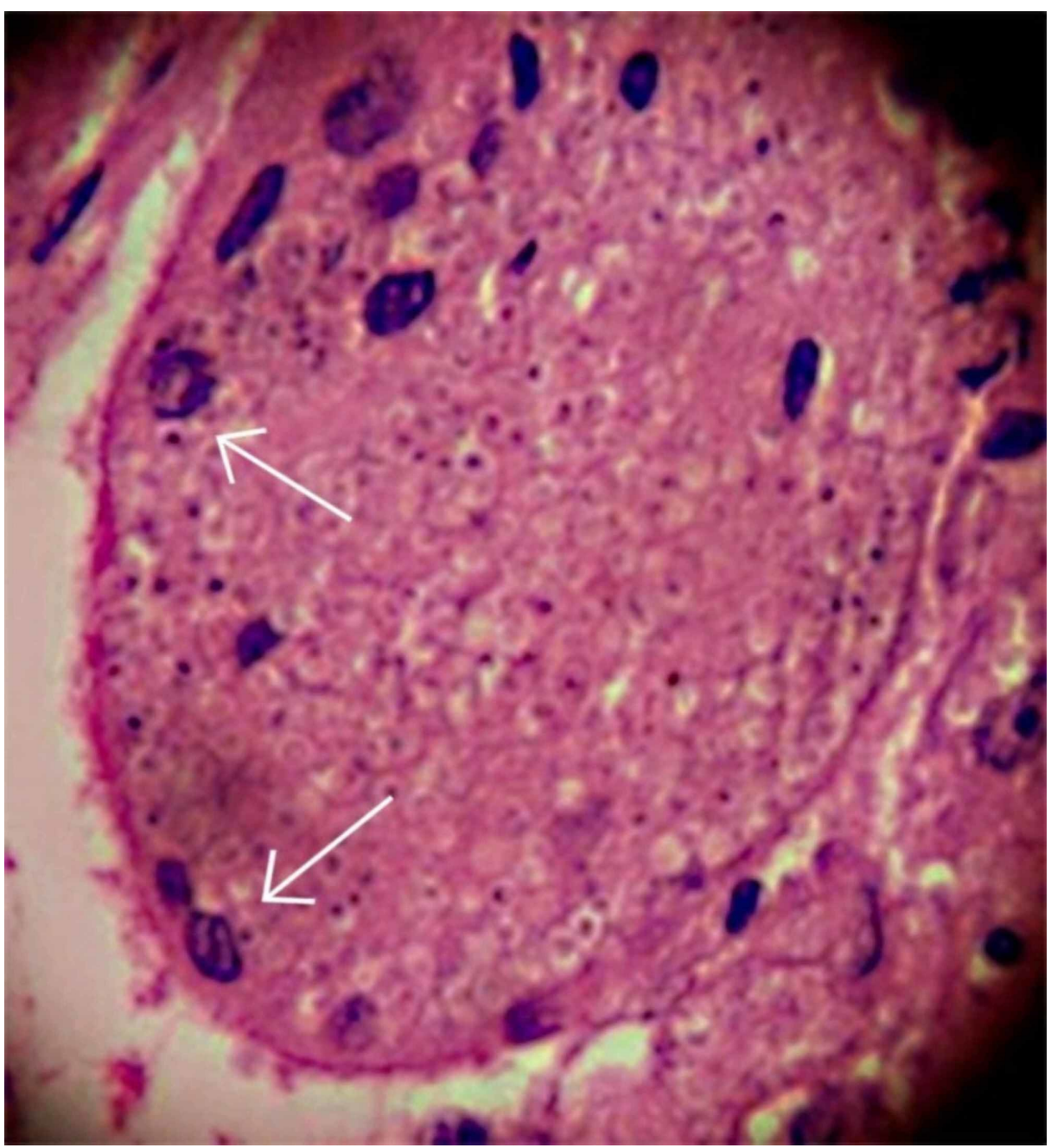

FIGURE 2: Histopathological biopsy study obtained from pseudotumoral mass in the sigmoid colon.

Histoplasma capsulatum highlighted with periodic acid-Schiff (PAS) stain (arrow). 
Amphotericin B was stopped after 14 days due to the improvement in the patient's symptoms and then switched to itraconazole $200 \mathrm{mg}$ every eight hours for four days, followed by $200 \mathrm{mg}$ twice daily for one year with the successful regression of the symptoms.

\section{Discussion}

This case report is unique because the infection presented as a pseudotumoral mass obstructing $80 \%$ of the colonic lumen. Although we identified six cases of intestinal $H$. capsulatum in immunosuppressed patients reported in the literature, all of those cases presented as intestinal ulcers rather than obstructive entities [27].

Histoplasmosis is a common comorbidity in AIDS patients [8]; however, this condition can also be reported in immunocompetent patients $[4,9]$.

The infection is caused by breathing spores from soil contaminated by bird and bat droppings $[1,4,9]$. For this reason, pulmonary histoplasmosis is the classical presentation of the infection. Other organs like the skin and adrenal glands can also be affected. The gastrointestinal system can be affected in the disseminated form of the disease [1].

In our patient, the diagnosis of intestinal histoplasmosis was incidental because there was no preceding pulmonary presentation which usually leads to a suspicion of a disseminated condition.

Some cases in the literature report diarrhea and abdominal pain as the most common symptoms [2-7]; however, our patient presented with chronic constipation. This atypical symptom may be the result of the mechanical obstruction of the lumen by the mass.

The detection of urine H. capsulatum antigens is used in the diagnosis of the pulmonary infection and the disseminated presentation $[1,8,10]$.

Intestinal ulcers are common colonoscopic findings in gastrointestinal histoplasmosis [2, 4-7]. An obstructive lesion was reported only by Winn et al. [4].

\section{Conclusions}

We presented a rare case of intestinal histoplasmosis by H. capsulatum presented in the form of a pseudotumoral obstructive mass. This report raises awareness to consider this pathology as a new differential diagnosis in patients with AIDS due to the increase in atypical presentations of infections.

\section{Additional Information \\ Disclosures}

Human subjects: Consent was obtained by all participants in this study. Conflicts of interest: In compliance with the ICMJE uniform disclosure form, all authors declare the following: Payment/services info: All authors have declared that no financial support was received from any organization for the submitted work. Financial relationships: All authors have declared that they have no financial relationships at present or within the previous three years with any organizations that might have an interest in the submitted work. Other relationships: All authors have declared that there are no other relationships or activities that could appear to have influenced the submitted work.

\section{References}

1. Caceres DH, Knuth M, Derado G, et al.: Diagnosis of progressive disseminated histoplasmosis in advanced HIV: a meta-analysis of assay analytical performance. J Fungi. 2019, 5:76. 10.3390/jof5030076

2. Spinner MA, Paulin HN, Wester CW: Duodenal histoplasmosis presenting with upper gastrointestinal bleeding in an AIDS patient. Case Rep Gastrointest Med. 2012, 2012:515872. 10.1155/2012/515872

3. Escobar B, Maldonado VN, Ansari S, et al.: Antigen negative gastrointestinal histoplasmosis in an AIDS patient. Am J Case Rep. 2014, 15:90-93. 10.12659/AJCR.889940

4. Winn JN, Akbar S, Theivanayagam S, et al.: Disseminated histoplasmosis presenting as colonic pseudotumor. Endoscopy. 2013, 45:E155-E156.

5. Alva E, Vásquez J, Frisancho O, et al.: [Colonic histoplasmosis as a diagnostic manifestation of AIDS] . Rev Gastroenterol Perú. 2010, 30:163-166.

6. Bhinder J, Mori A, Cao W, et al.: A case of isolated gastrointestinal histoplasmosis . Cureus. 2018, 10:2951. 10.7759/cureus.2951

7. Zarrinpar A, Lin GY, Lonergan JT: A rare cause of an ileocecal fistula in an AIDS patient. Gastrointestinal infection by Histoplasma capsulatum infection identified with internal transcribed spacer primer sets. Gastroenterology. 2013, 144:857-858. 10.1053/j.gastro.2012.11.030

8. Nacher M, Blanchet D, Bongomin F, et al.: Histoplasma capsulatum antigen detection tests as an essential diagnostic tool for patients with advanced HIV disease in low and middle income countries: a systematic 


\section{Cureus}

review of diagnostic accuracy studies. PLOS Negl Trop Dis. 2018, 12:0006802. 10.1371/journal.pntd.0006802

9. Yang B, Lu L, Li D, et al.: Colonic involvement in disseminated histoplasmosis of an immunocompetent adult: case report and literature review. BMC Infect Dis. 2013, 13:143. 10.1186/1471-2334-13-143

10. Torres-González P, Niembro-Ortega MD, Martínez-Gamboa A, et al.: Diagnostic accuracy cohort study and clinical value of the histoplasma urine antigen (ALPHA Histoplasma EIA) for disseminated histoplasmosis among HIV infected patients: a multicenter study. PLOS Negl Trop Dis. 2018, 12:0006872.

10.1371/journal.pntd.0006872 